\title{
Ekiden to iPS Cells
}

\author{
Shinya Yamanaka
}

When I started a long ekiden - a relay marathon by multiple runners-toward cellular reprogramming about a decade ago, not many teams joined the start of the race. At the time, many laboratories were trying to differentiate embryonic stem (ES) cells into various functional cells, inspired by the report on the isolation of human ES cells ${ }^{1}$. In contrast, I tried to dedifferentiate somatic cells back to the embryonic state. I was not sure if we could reach our goal in my lifetime, but thanks to diligent colleagues and students in my laboratory (Fig. 1), luck favored us.

In August 2006, we announced the generation of ES cell-like stem cells by introducing four transcription factors-Oct3/4, Sox2, Klf4 and c-Myc - into mouse fibroblasts by retroviral vectors ${ }^{2}$. I named this new type of cells 'induced pluripotent stem (iPS) cells'. We reported the generation of human iPS cells ${ }^{3}$ on 20 November 2007, the same day that James Thomson and his colleagues also announced their success in making human iPS cells ${ }^{4}$.

With the ability to differentiate into virtually all types of cells and to grow robustly like ES cells, iPS cells have enormous potential for pharmaceutical and clinical applications ${ }^{5,6}$. The technology is expected to be used for generating disease models, drug screening, toxicology and regenerative medicine, circumventing two obstacles surrounding ES cells - the ethical controversy over destruction of embryos to isolate ES cells and the risks of immune rejection when ES-derived cells are transplanted into a person. In vitro uses of iPS cells are just around the corner, but there are still many hurdles before their clinical application. However, I believe that strenuous efforts of researchers around the world will make the promises a reality in the not-too-distant future.

Shinya Yamanaka is at the Center for iPS Cell Research and Application, Kyoto University, Kyoto, Japan and the Gladstone Institute of Cardiovascular Disease, San Francisco, California, USA.

e-mail: yamanaka@cira.kyoto-u.ac.jp

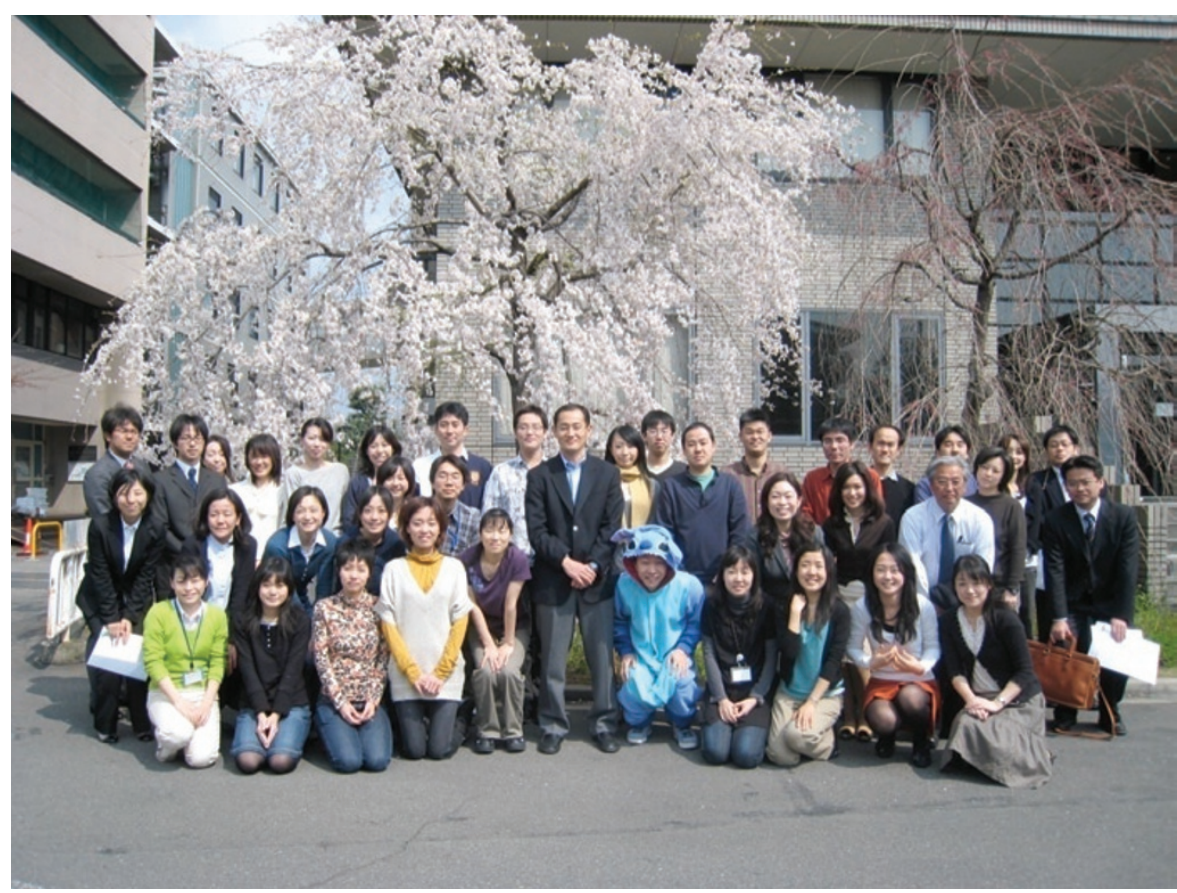

Figure 1 Members of Yamanaka's lab and the Center for iPS Cell Research and Application by a cherry tree blooming at Kyoto University, Japan, in April 2009.

Today, iPS cell research has become one of the hottest areas in the life sciences, with an increasing number of laboratories worldwide working on the technology. Many new findings have been reported in the short time since the first report was published ${ }^{5,7}$. I cannot stress enough that without the many studies using mouse and human ES cells that have appeared since the isolation of mouse ES cells in 1981 (refs. 8,9), we would not have been able to generate iPS cells and advance the field at such rapid speed. In fact, my encounter with mouse ES cells led me to start the ekiden toward iPS cell generation.

\section{From orthopedic surgeon to scientist}

I started my professional career as an orthopedic surgeon at a hospital in Osaka, western Japan, after graduating from Kobe University in 1987. During my residency, however, I real- ized that I was not talented in surgery, and that even talented surgeons cannot cure many diseases and injuries, so I became interested in basic medical sciences. In 1989, I started my marathon as a scientist by becoming a $\mathrm{PhD}$ student in pharmacology at Osaka City University Graduate School of Medicine, working under Kenjiro Yamamoto. In the next four years, I learned the essentials about how to design, conduct and analyze experiments from my direct mentor, Katsuyuki Miura. As a PhD student in pharmacology, I was astonished by mouse transgenesis and gene targeting, which specifically induce or delete a gene of interest, because no pharmacological agents could perform such miracles. After finishing my PhD work in 1992 (ref. 10), I applied for as many postdoctoral positions as I could in labs doing mouse molecular genetics. However, it was very natural that a failed 
surgeon with little experience in molecular biology had a hard time finding a position.

A turning point came when I got a fax from Thomas Innerarity at the Gladstone Institute of Cardiovascular Diseases in San Francisco (Fig. 2). After a short telephone conversation, Tom was brave enough to give me a position! When I joined his lab, he had a promising hypothesis that forced expression of APOBEC1, the ApoB messenger RNA editing enzyme, in liver would lower plasma cholesterol levels and thus prevent atherosclerosis. To test this hypothesis, we generated transgenic mice overexpressing Apobec 1 in their livers. To our surprise, however, the transgenic mice developed liver tumors ${ }^{11}$. We learned the hard way that Apobec1 is a potent proto-oncogene. Naturally, we were disappointed, but at the same time we became very interested in the molecular mechanisms of this totally unexpected finding. We identified a novel target of Apobec1, Nat1, which was aberrantly edited in the transgenic mouse livers $^{12}$.

\section{Encounter with ES cells and PAD}

I decided to generate Nat1-knockout mice to study the gene's function. Robert Farese at Gladstone and his research associate Heather Myers kindly taught me how to culture mouse ES cells and make chimeras. But, in 1996, I had to reluctantly go back to Japan because of a family situation. Before I left Gladstone, a European postdoc at the institute said to

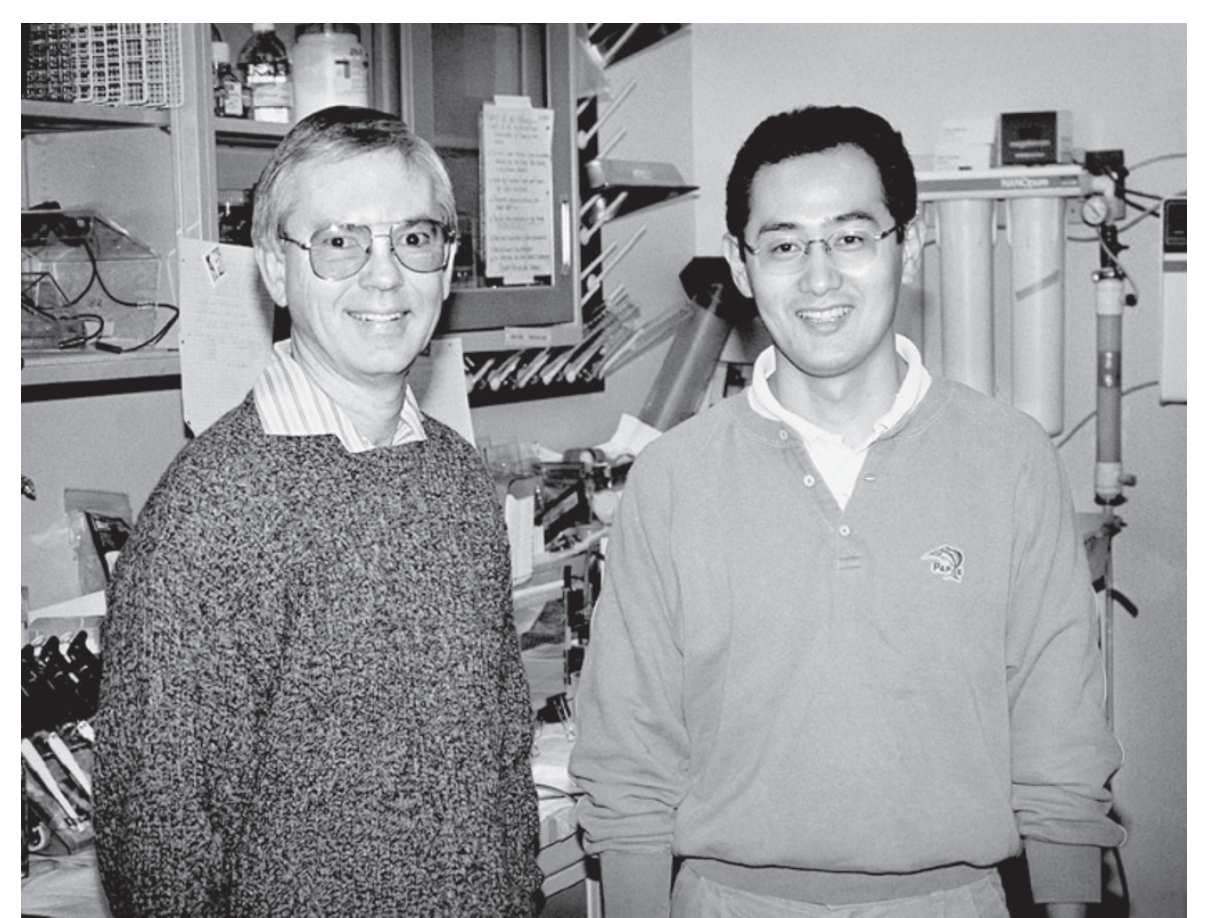

Figure 2 Thomas L. Innerarity and Shinya Yamanaka at the Gladstone Institute. Photo courtesy of the

J. David Gladstone Institutes.

me, "Shinya, be careful of PAD, post-America depression!” At that time, I could not understand what he meant.

Back in Japan, I got a position as an assistant professor in the department of pharmacology at Osaka City University. Tom let me continue the Nat1 work and sent three chimeric mice to Japan. The chairman of the department, Hiroshi Iwao, was very supportive and allowed me to work on Nat1, which seemed to have little value in pharmacology. I found that Nat1 is required for early mouse development ${ }^{13}$. More importantly, I found that Nat1-null ES cells proliferate normally but cannot properly differentiate. This unexpected finding changed the meaning of mouse ES cells to me from research tool to research subject. I became interested in how ES cells maintain their differentiation ability while rapidly proliferating.

At the same time, however, I discovered that I suffered from PAD. At the medical school, very few scientists showed interest in Nat 1 or in the basic biology of mouse ES cells. I was advised to work on something more related to medicine. I could not get good enough funding and had to change cages of numerous mice by myself every week. The Nat1 work was being rejected by many journals. I felt lonely and depressed, and I was about to stop running in the marathon as a scientist.

Fortunately, two events rescued me from PAD and from giving up on science. First, Thomson and his colleagues announced the

\section{Induced pluripotent stem (iPS) cells}

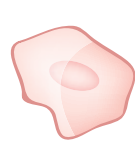

Fibroblasts

Mouse iPS

cells in 2006

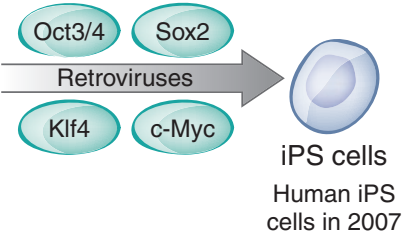

Figure 3 Induced pluripotent stem (iPS) cells can be established by introducing genes encoding four transcription factors-Oct3/4, Sox2, KIf4 and c-Myc_into fibroblasts.

generation of human ES cells in 1998 (ref. 1). His success taught me that ES cells have enormous potential in medicine and encouraged me to continue running in the scientific marathon. Second, in December 1999, I got a new position as associate professor-with my own laboratory for the first time-at the Nara Institute of Science and Technology (NAIST). This institute has many good investigators in basic and applied sciences, an excellent research environment and good PhD students. I was lucky enough to have several talented colleagues and students in my laboratory. It was then that my scientific marathon became an ekiden, a relay by several runners.

\section{Ekiden at NAIST toward iPS cell generation}

I was expected to establish a knockout mouse core facility at NAIST. It was a difficult task, but thanks to an excellent technician, Tomoko Ichisaka, and to funding from NAIST, we were able to establish it within a few years. The first gene that we knocked out was Fbxo15, which we identified as a gene specifically expressed in mouse ES cells. One of my first PhD students, Yoshimi Tokuzawa, with the help of Tomoko, successfully targeted the gene ${ }^{14}$. However, we did not see any phenotypes in mice or ES cells lacking Fbxo15. We were disappointed, but this very first knockout mouse line turned out later to be useful in the generation of iPS cells.

As a principal investigator, I needed to set a long-term goal for my laboratory. Because of my interest in ES cells, because of the successful generation of human ES cells and because I had to use ES cells anyway in the knockout mouse core, I decided to list "ES cells" in the title of my website. To overcome two major hurdles of human ES cells-ethical issues regarding the use of human embryos and immune rejection after transplantation-I decided nuclear reprogramming would be the goal of our ekiden. More precisely, we started 


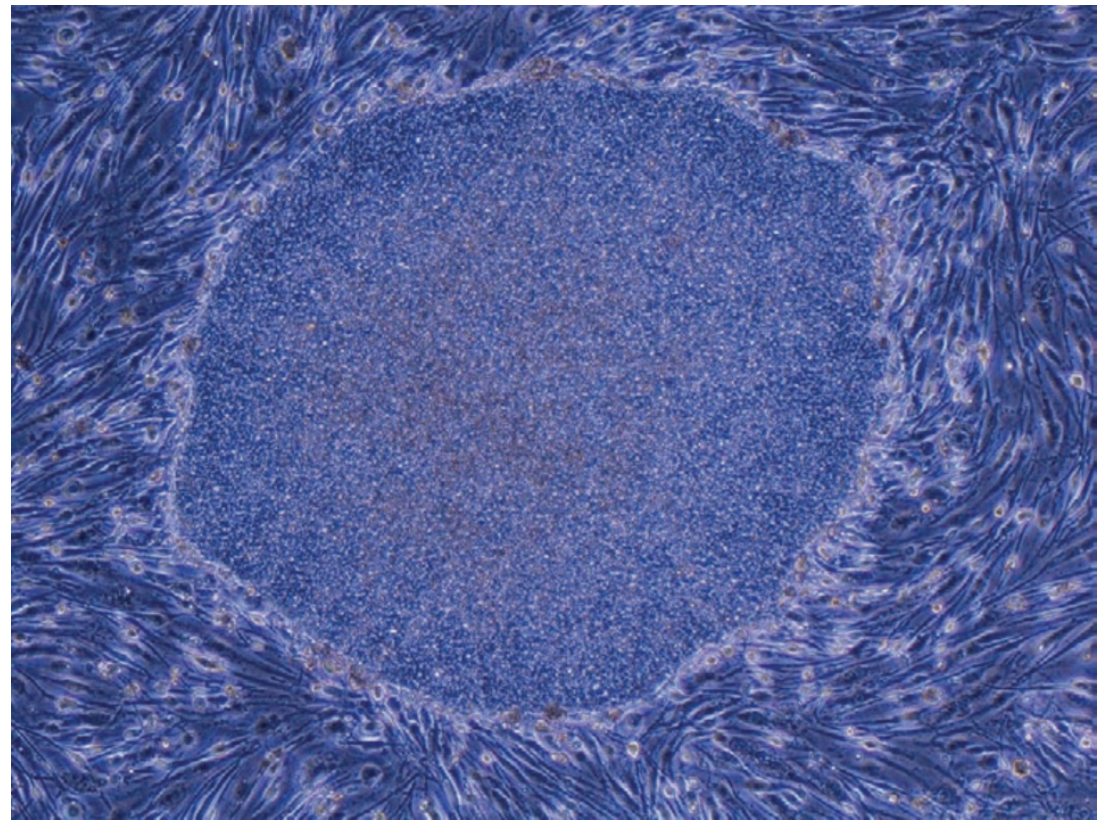

Figure 4 iPS colony generated from adult human dermal fibroblasts by introducing the four factors using retroviral vectors.

a project to generate ES cell-like pluripotent stem cells directly from somatic cells.

The concept of cellular reprogramming was established a long time ago. In 1962, the year I was born, Sir John Gurdon reported the generation of new frogs by transferring tadpole intestine cell nuclei into enucleated eggs from the African clawed toad, Xenopus laevis ${ }^{15}$. Dolly the sheep, reported in 1997, was another example ${ }^{16}$, as was a 2001 report showing that thymocytes acquire pluripotency upon electrofusion with mouse ES cells ${ }^{17}$. These experiments clearly showed that eggs and ES cells contain factors that induce totipotency or pluripotency in somatic cells.

I first hypothesized that factors that maintain pluripotency of mouse ES cells might induce pluripotency in somatic cells. With the great help of the initial members of my lab-Tomoko, Yoshimi, two other PhD students (Kazutoshi Takahashi and Eiko Kaiho) and an assistant (Kaoru Mitsui) — we identified many factors that either are specifically expressed by or have important roles in mouse ES cells ${ }^{14,18-22}$. Among them was the transcription factor Klf4, identified by Yoshimi. Between our own work and that of other groups, by 2004 we had collected 24 initial candidates that might be able to induce pluripotency. We then needed a simple and sensitive assay system to evaluate these candidates, and the Fbxo15-knockout mice turned out to be such a system ${ }^{14}$. Instead of simply deleting the gene, we knocked the neomycin resistant gene $\left(n e o^{\mathrm{R}}\right)$ into the Fbxo15 locus.
Somatic cells derived from these mice do not express $n e o^{\mathrm{R}}$ and are sensitive to the antibiotic G418. Somatic cells that become ES cell-like after transfection with some of our candidate genes should express $n e o^{\mathrm{R}}$ and become resistant to G418.

\section{Last surge in Kyoto toward iPS cell generation}

In 2004, I moved to Kyoto University with the 24 candidate genes, the Fbxo15-neo ${ }^{\mathrm{R}}$ knockin mice and many members of my lab, including Tomoko and Kazutoshi. I asked Kazutoshi to test the 24 candidates using the Fbxo15 knock-in mice. He was pleased to take over this very risky project and did an excellent job as the last runner in the ekiden. When Kazutoshi introduced each candidate into the $F b x o 15-n e o^{\mathrm{R}}$ reporter fibroblasts using retroviral vectors, no G418-resistant colonies emerged. However, when he introduced the mixture of all 24 retroviruses, we observed several drug-resistant colonies in a petri dish $^{2}$. These cells were similar to ES cells in morphology, proliferation and gene expression. When transplanted into nude mice, they formed teratomas containing a variety of tissues from all three germ layers, showing their pluripotency. Among the myriad combinations of the 24 factors, Kazutoshi found that four transcription factors-Oct3/4, Sox2, Klf4 and c-Myc-are essential (Fig. 3).

We termed these cells 'iPS cells'. By improving the induction and selection methods, Keisuke Okita, with the help of Tomoko, succeeded in making iPS cells that are competent for production of adult chimeras and germline transmission ${ }^{23}$. Masato Nakagawa and Michiyo Koyanagi then showed that iPS cells can be generated without c-Myc ${ }^{24}$. Takashi Aoi showed that iPS cells can be generated not only from fibroblasts, but also from adult mouse hepatocytes and gastric epithelial cells ${ }^{25}$. Kazutoshi and Koji Tanabe then succeeded in making iPS cells from human adult skin fibroblasts with the same four factors $^{3}$ (Fig. 4).

\section{Just the beginning of a new ekiden}

The potential of the iPS cell technology in medicine, drug discovery, toxicology and animal technologies is enormous ${ }^{26}$ (Fig. 5). At the same time, the technology is still in its infancy, and there are many challenges

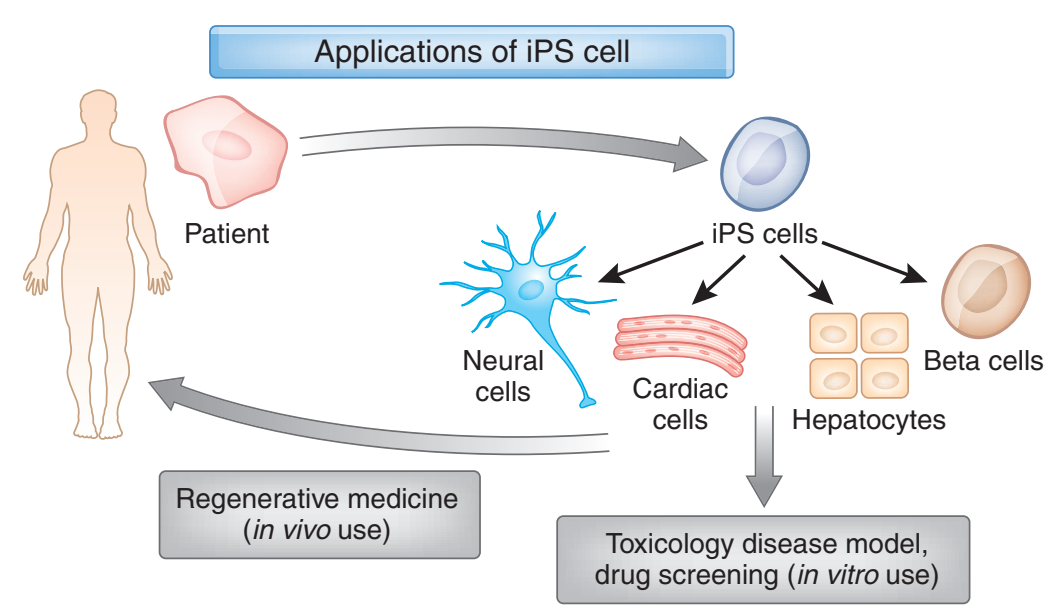

Figure 5 Possible applications of iPS cells. iPS cells generated from somatic cells of patients with intractable diseases can be differentiated into the affected cell types and used to study disease mechanisms. The cells can also be used as tools in drug discovery and to develop cell therapies. 
to be overcome before the various applications can be used successfully. We have just started the new ekiden toward application of the iPS cell technology. Owing to its simple and reproducible method, numerous laboratories around the world have started running in the ekiden. It may take many years to reach the goal, but, as a scientist who used to be a physician and was not able to help patients, I sincerely hope the technology will contribute to the development of new cures for people suffering from various diseases and injuries.

\section{ACKNOWLEDGMENTS}

I would like to thank my father, who passed away 20 years ago, for instructing me to be a physician; $m y$ mother and sister for their encouragement; and my wife and daughters for their continuous support. In addition to those mentioned in this commentary, many colleagues and students run the ekiden; without their efforts, we would not have been able to generate iPS cells. I am grateful to A. Nakamura for her aid in writing this commentary and to the many members of the Center for iPS Cell Research and Application and Gladstone Institute for their technical and administrative support.

1. Thomson, J.A. et al. Science 282, 1145-1147 (1998).

2. Takahashi, K. \& Yamanaka, S. Cell 126, 663-676 (2006).

3. Takahashi, K. et al. Cell 131, 861-872 (2007).

4. Yu, J. et al. Science 318, 1917-1920 (2007).

5. Yamanaka, S. Cell 137, 13-17 (2009).

6. Yamanaka, S. Cell Stem Cell 1, 39-49 (2007).

7. Yamanaka, S. Nature 460, 49-52 (2009).

8. Martin, G.R. Proc. Natl. Acad. Sci. USA 78, 7634 7638 (1981).

9. Evans, M.J. \& Kaufman, M.H. Nature 292, 154-156 (1981).

10. Yamanaka, S., Miura, K., Yukimura, T., Okumura, M. \& Yamamoto, K. Circ. Res. 70, 893-901 (1992).

11. Yamanaka, S. et al. Proc. Natl. Acad. Sci. USA 92 8483-8487 (1995).
12. Yamanaka, S., Poksay, K.S., Arnold, K.S. \& Innerarity, T.L. Genes Dev. 11, 321-333 (1997).

13. Yamanaka, S. et al. EMBO J. 19, 5533-5541 (2000).

14. Tokuzawa, Y. et al. Mol. Cell. Biol. 23, 2699-2708 (2003).

15. Gurdon, J.B. J. Embryol. Exp. Morphol. 10, 622-640 (1962).

16. Wilmut, I., Schnieke, A.E., McWhir, J., Kind, A.J. \& Campbell, K.H. Nature 385, 810-813 (1997).

17. Tada, M., Takahama, Y., Abe, K., Nakatsuji, N. \& Tada, T. Curr. Biol. 11, 1553-1558 (2001).

18. Tsubooka, N. et al. Genes Cells 14, 683-694 (2009).

19. Imamura, M. et al. BMC Dev. Biol. 6, 34 (2006).

20. Maruyama, M., Ichisaka, T., Nakagawa, M. \& Yamanaka, S. J. Biol. Chem. 280, 24371-24379 (2005).

21. Takahashi, K., Mitsui, K. \& Yamanaka, S. Nature 423 541-545 (2003)

22. Mitsui, K. et al. Cell 113, 631-642 (2003).

23. Okita, K., Ichisaka, T. \& Yamanaka, S. Nature 448 313-317 (2007).

24. Nakagawa, M. et al. Nat. Biotechnol. 26, 101-106 (2008).

25. Aoi, T. et al. Science 321, 699-702 (2008).

26. Nishikawa, S., Goldstein, R.A. \& Nierras, C.R. Nat. Rev. Mol. Cell Biol. 9, 725-729 (2008). 